\title{
Electromagnetic and material contributions to stress, energy, and momentum in metamaterials
}

\author{
Brandon A. Kemp ${ }^{1}$ and Cheyenne J. Sheppard ${ }^{2}$ \\ ${ }^{1}$ College of Engineering, Arkansas State University, Jonesboro, AR, USA \\ ${ }^{2}$ College of Sciences and Mathematics, Arkansas State University, Jonesboro, AR, USA \\ *corresponding author, E-mail: bkemp@astate.edu
}

\begin{abstract}
We demonstrate modeling of the field-kinetic and material response subsystem for various media and extend the models to dispersive negative index metamaterials. It is shown that neither the Minkowski or Abraham models are universally correct, as demonstrated to describe metamaterials under both the field-kinetic and wave SEM models for various applications such as negative refraction, perfect lensing, and invisibility cloaking.
\end{abstract}

\section{Introduction}

Electromagnetic momentum has been debated since the early $20^{\text {th }}$ century. The so-called Abraham-Minkowski debate is attributed to two independent stress-energymomentum (SEM) tensors postulated for mathematically modeling electrodynamics of media [1]. Significant attention has been given to the momentum density expressions associated with the rival SEM tensors. The momentum density expressions were defined as being either $\overline{\mathcal{D}} \times \overline{\mathcal{B}}$ from the Minkowski tensor or $\epsilon_{0} \mu_{0} \overline{\mathcal{E}} \times \overline{\mathcal{H}}$ from the Abraham tensor, where $\overline{\mathcal{D}}$ and $\overline{\mathcal{B}}$ represent the electric displacement and magnetic induction fields, $\overline{\mathcal{E}}$ and $\overline{\mathcal{H}}$ are the electric and magnetic fields, and $\epsilon_{0}$ and $\mu_{0}$ are the permittivity and permeability of vacuum, respectively. Barnett presented a resolution to the photon momentum controversy in 2010, which related the Abraham density to the kinetic momentum of light and the Minkowski density to the canonical momentum of light [2]. However, the original debate was in regard to the relativistic invariance of the $4 \times 4$ SEM tensors; Barnett's partial resolution only identified the kinetic and canonical momentum densities.

Three other well-known SEM tensors have been proposed over the past century, and have found significant use in scientific and engineering modeling applications [3]. First, the Einstein-Laub tensor, like the Abraham and Minkowski tensors, utilizes the Minkowski fields, and it shares the Abraham momentum density. Second, the Amperian or "Lorentz" tensor derives from the Amperian formulation or $E B$-representation and utilizes the Amperian fields. Third, the Chu formulation or $E H$-representation was developed in the 1960's, and the Chu SEM tensor utilizes the Chu fields. It is important to note that the latter two formulations uniquely define the fields within matter, while the Abraham, Minkowski, and Einstein-Lab tensors all use the common Minkowski fields.

In this correspondence, we demonstrate modeling of the field-kinetic and material response subsystem for dielectric media and extend the models to negative index metamaterials, which necessarily include dispersion and loss. In this model, we consider the total SEM tensor as a conservation of energy and momentum such that

$$
\begin{gathered}
\mathfrak{T}_{\text {total }}=\mathfrak{T}_{\text {mech }}+\mathfrak{T}_{\text {can }}=\mathfrak{T}_{\text {mech }}+\mathfrak{T}_{\text {mat }}+\mathfrak{T}_{F_{k}}, \\
\mathfrak{T}_{F_{k}}+\mathfrak{T}_{\text {mat }} \neq \mathfrak{T}_{\text {Min }}
\end{gathered}
$$

where $\mathfrak{T}_{\text {mech }}$ represents an external mechanical input of work, $\mathfrak{T}_{m a t}$ is the material response subsystem, and $\mathfrak{T}_{F_{k}}$ is the field-kinetic subsystem. We show that the fieldkinetic subsystem as given by the Chu formulation represents the energy and momentum contained within the fields [4], and the sum of the field-kinetic and material response subsystems represent the wave or canonical subsystem [5], which reduces to the Minkowski subsystem only under negligible dispersion [6]. Our conclusion is that neither the Minkowski or Abraham models are universally correct. We demonstrate our view applied to the physics of negative index materials under both the field-kinetic and canonical SEM models for applications such as negative refraction, which is fundamental to perfect lensing and invisibility cloaking. First, we review the mathematical framework of SEM tensors, relativistic invariance requirements, and applicability to electrodynamics in Section 2. Second, the field-kinetic and canonical SEM tensors are determined for a broad class of causal, isotropic media in Section 3. Then, we discuss the implications of negative refraction in Section 4. Conclusions are presented in Section 5.

\section{SEM Tensors}

Energy and momentum continuity for a given system can be divided into subsystems and mathematically represented as $[1,3]$

$$
\begin{aligned}
\varphi_{j}(\bar{r}, t) & =-\nabla \cdot \bar{S}_{j}(\bar{r}, t)-\frac{\partial W_{j}(\bar{r}, t)}{\partial t} \\
\bar{f}_{j}(\bar{r}, t) & =-\nabla \cdot \overline{\bar{T}}_{j}(\bar{r}, t)-\frac{\partial \bar{G}_{j}(\bar{r}, t)}{\partial t}
\end{aligned}
$$

where $\bar{f}_{j}$ is the force density, $\varphi_{j}$ is the power density, $\overline{\bar{T}}_{j}$ is the momentum flux or stress tensor, $\bar{S}_{j}$ is the power flux, 
$\bar{G}_{j}$ is the momentum density, and $W_{j}$ is the energy density. Each $j$ may represent any subsystem. Closing the overall system, such that $\sum_{j} \bar{f}_{j}=0$ and $\sum_{j} \varphi_{j}=0$ indicates momentum and energy conservation, respectively.

The choice of which momentum density, stress tensor, and force density are applied in any given problem are inherently tied to the formulation of electrodynamics being considered and the interpretation rendered. It is impossible to sidestep the momentum controversy by employing a Lorentz force density since, as the theoretical construction implies, each force density is tied to a momentum density and stress tensor through a formulation of Maxwell's equations [3]. One may rearrange the continuity equations, but such mathematical exercises should not be taken as reason for interpretation [4, 7]. For reference, Table 1 lists the leading electromagnetic momentum densities and stress tensors from the literature. The corresponding force densities for the stationary [1] and fully relativistic formulations [3] can also be found in the literature.

Table 1: Leading electromagnetic momentum densities and stress tensors.

\begin{tabular}{ccc}
\hline & $\bar{G}$ & $\bar{T}$ \\
\hline Abr. & $\epsilon_{0} \mu_{0} \overline{\mathcal{E}} \times \overline{\mathcal{H}}$ & $\frac{1}{2}(\overline{\mathcal{D}} \cdot \overline{\mathcal{E}}+\overline{\mathcal{B}} \cdot \overline{\mathcal{H}}) \overline{\bar{I}}-\overline{\mathcal{D}} \overline{\mathcal{E}}-\overline{\mathcal{B}} \overline{\mathcal{H}}$ \\
Min. & $\overline{\mathcal{D}} \times \overline{\mathcal{B}}$ & $\frac{1}{2}(\overline{\mathcal{D}} \cdot \overline{\mathcal{E}}+\overline{\mathcal{B}} \cdot \overline{\mathcal{H}}) \overline{\bar{I}}-\overline{\mathcal{D}} \overline{\mathcal{E}}-\overline{\mathcal{B}} \overline{\mathcal{H}}$ \\
Amp. & $\epsilon_{0} \overline{\mathcal{E}} \times \overline{\mathcal{B}}$ & $\frac{1}{2}\left(\epsilon_{0} \overline{\mathcal{E}} \cdot \overline{\mathcal{E}}+\mu_{0}^{-1} \overline{\mathcal{B}} \cdot \overline{\mathcal{B}}\right) \overline{\bar{I}}-\epsilon_{0} \overline{\mathcal{E}} \overline{\mathcal{E}}-\mu_{0}^{-1} \overline{\mathcal{B}} \overline{\mathcal{B}}$ \\
E-L & $\epsilon_{0} \mu_{0} \overline{\mathcal{E}} \times \overline{\mathcal{H}}$ & $\frac{1}{2}\left(\epsilon_{0} \overline{\mathcal{E}} \cdot \overline{\mathcal{E}}+\mu_{0} \overline{\mathcal{H}} \cdot \overline{\mathcal{H}}\right) \overline{\bar{I}}-\overline{\mathcal{D}} \overline{\mathcal{E}}-\overline{\mathcal{B}} \overline{\mathcal{H}}$ \\
Chu & $\epsilon_{0} \mu_{0} \overline{\mathcal{E}} \times \overline{\mathcal{H}}$ & $\frac{1}{2}\left(\epsilon_{0} \overline{\mathcal{E}} \cdot \overline{\mathcal{E}}+\mu_{0} \overline{\mathcal{H}} \cdot \overline{\mathcal{H}}\right) \overline{\bar{I}}-\epsilon_{0} \overline{\mathcal{E}} \overline{\mathcal{E}}-\mu_{0} \overline{\mathcal{H}} \overline{\mathcal{H}}$ \\
\hline
\end{tabular}

As one considers the amount of work being done on the system, one also sees that the momentum and stress are explicitly tied to the energy density and flux. This can be illustrated using the work-energy relation or the Relativistic Principle of Virtual Power (RPVP) [4, 5]. In Section 2.2 it will be demonstrated, instead, using a straightforward application of the Lorentz transformation. For now, we list for reference in Table 2 the leading electromagnetic energy densities and power flows corresponding to the momentum quantities in Table 1.

Table 2: Leading electromagnetic energy densities and power flows.

\begin{tabular}{lcc}
\hline & $\bar{S}$ & $W$ \\
\hline Abr. & $\overline{\mathcal{E}} \times \overline{\mathcal{H}}$ & $\frac{1}{2}(\overline{\mathcal{D}} \cdot \overline{\mathcal{E}}+\overline{\mathcal{B}} \cdot \overline{\mathcal{H}})$ \\
Min. & $\overline{\mathcal{E}} \times \overline{\mathcal{H}}$ & $\frac{1}{2}(\overline{\mathcal{D}} \cdot \overline{\mathcal{E}}+\overline{\mathcal{B}} \cdot \overline{\mathcal{H}})$ \\
Amp. & $\mu_{0}^{-1} \overline{\mathcal{E}} \times \overline{\mathcal{B}}$ & $\frac{1}{2}\left(\epsilon_{0} \overline{\mathcal{E}} \cdot \overline{\mathcal{E}}+\mu_{0}^{-1} \overline{\mathcal{B}} \cdot \overline{\mathcal{B}}\right)$ \\
E-L & $\overline{\mathcal{E}} \times \overline{\mathcal{H}}$ & $\frac{1}{2}\left(\epsilon_{0} \overline{\mathcal{E}} \cdot \overline{\mathcal{E}}+\mu_{0} \overline{\mathcal{H}} \cdot \overline{\mathcal{H}}\right)$ \\
Chu & $\overline{\mathcal{E}} \times \overline{\mathcal{H}}$ & $\frac{1}{2}\left(\epsilon_{0} \overline{\mathcal{E}} \cdot \overline{\mathcal{E}}+\mu_{0} \overline{\mathcal{H}} \cdot \overline{\mathcal{H}}\right)$ \\
\hline
\end{tabular}

The continuity equations can be rewritten in fourdimensional coordinates $(\bar{r}, i c t)$ so that

$$
\begin{aligned}
\bar{f}_{j} & =\square \cdot\left[\overline{\mathcal{T}}_{j},-i c \bar{G}_{j}\right] \\
-i \frac{\varphi_{j}}{c} & =\square \cdot\left[-i \frac{\bar{S}_{j}}{c}, W_{j}\right]
\end{aligned}
$$

so that $\square \cdot \overline{\overline{\mathfrak{T}}}_{j}=\overline{\mathfrak{F}}_{j}$, where $\square=[\nabla, \partial / \partial(i c t)], \overline{\mathfrak{F}}_{j}=$ $\left[-\bar{f}_{j},-i \varphi_{j} / c\right]$, and the SEM tensor is

$$
\overline{\overline{\mathfrak{T}}}_{j}=\left[\begin{array}{cc}
\overline{\overline{\mathcal{T}}}_{j} & -i c \bar{G}_{j} \\
-\frac{i}{c} \bar{S}_{j} & W_{j}
\end{array}\right] .
$$

Here, we have defined $\overline{\overline{\mathcal{T}}}_{j} \equiv-\overline{\bar{T}}_{j}$.

\subsection{Equivalence of total force}

The electromagnetic force density $\bar{f}$ and power density $\varphi$ inside an object depend upon formulation applied. In general, the equations take the form of Eqs. (3), where the force densities are defined by the corresponding momentum density $\bar{G}$ and stress tensor $\overline{\bar{T}}$ given in Table 1 and the power densities $\varphi$ are defined by the corresponding energy density $W$ and power flux $\bar{S}$ given in Table 2. The total electromagnetic force $\bar{F}(t)$ on an object can be computed by integrating an electromagnetic force density $\bar{f}(\bar{r}, t)$ over the volume $V$ of the object. An exact, mathematically equivalent approach is to apply the divergence theorem to reduce the contribution of an electromagnetic stress tensor $\overline{\bar{T}}$ to an integral over the surface $A$ with outward pointing area element $d \bar{A}$ enclosing the volume $V$ so that the total force is calculated equivalently by

$$
\bar{F}(t)=-\int_{V} d V \frac{\partial}{\partial t} \bar{G}(\bar{r}, t)-\oint_{A} d \bar{A} \cdot \overline{\bar{T}}(\bar{r}, t) .
$$

In some cases, the total force may be equivalent between formulations although the force densities differ. The same argument may be applied to the transfer of energy since the energy continuity equation takes the same form.

\subsubsection{Equivalence of Total Force}

Fig. 1 (a) depicts an object surrounded by vacuum illustrating which total force equations are equivalent at any point in time. Integrating the electromagnetic force density $\bar{f}(\bar{r}, t)$ over the volume enclosing the object as depicted by the dashed line yields the total force and is given by Eq. (6), where the tensor reduces to the unambiguous vacuum Maxwell stress tensor since the surface of integration is outside the material. However, the momentum density $\bar{G}$ still depends on the electromagnetic formulation applied. Therefore, any formulations which share a common momentum density will produce identical results for the total force on an object at all points in time. The implication is that the Chu, Einstein-Laub, and Abraham force densities are equivalent in terms of total force and the Amperian formulation differs only in the modeling of magnetic media. The Minkowski SEM tensor will yield a different time varying force for dielectric and magnetic media.

A second example is an object submerged inside another medium such as a dielectric fluid as shown in Fig. 1 (b). The total force remains the same for two formulations if their momentum densities are identical. However, we must clarify how the force is computed. The electromagnetic force is determined by volume integration of the 


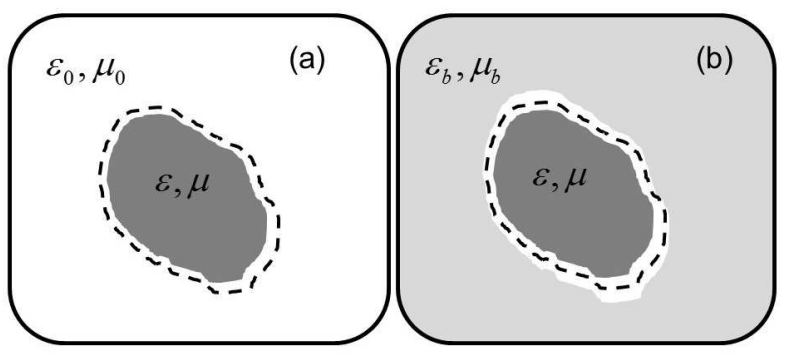

Figure 1: Illustration of total force calculation within a volume $V$ within the dashed line. The total force on (a) an object in vacuum is computed by volume integration of a force density within a region which completely encloses the material object and (b) an object embedded is computed in a similar way by considering a thin vacuum region between the two materials.

force density $\bar{f}(\bar{r}, t)$, and there will generally be both volume and surface forces at the boundary, the latter due to material discontinuities at the boundaries $[8,9]$. Any ambiguity as to which of the materials to which the surface forces should be assigned is removed by mathematically introducing a thin layer of vacuum separating the two materials. The volume integration is then applied to the submerged material. The tensor reduces to the vacuum stress tensor since the surface of the integration is in the vacuum region, and the forces applied to the two media are unambiguous. Taking the limit of the vacuum region to zero yields the unperturbed field problem with the surface forces applied correctly to the corresponding media [10]. In general, forces will be distributed to both the submerged object and the submerging fluid. The conclusion is unchanged. The total calculated time-domain force on an object is the same with any electromagnetic subsystem that share a common momentum density [3]. We will find that another conclusion from this thought exercise is important; once we identify the correct subsystem separation of field and matter it is necessary to assign a force on the embedded object and the embedding material $[10,11,12]$.

When materials are excited by monochromatic waves, the time-average force is generally the observable quantity of interest. The total average force on a material object due to time-harmonic fields is

$$
\langle\bar{F}\rangle=-\oint_{A} d \bar{A} \cdot\langle\overline{\bar{T}}\rangle
$$

where the momentum density does not contribute to the time-average force of time-harmonic fields [3].

The total average force is the integration of the average force density over a volume that includes the entire object. All such computations will yield identical results regardless of the force density applied. This statement is a result of the condition that all electromagnetic SEM tensors must be consistent with Maxwell's equations [1]. To illustrate this fact, one must only consider again the problem in Fig. 1 under time-averaged conditions. Since the momentum den- sity averages to zero, both computations in Fig 1 (a) and (b) reduce to the divergence of the vacuum stress tensor and the force is unambiguously independent of the formulation applied $[3,5]$. As a result, it is generally not possible to determine the time-domain force or energy equations from only the time-average quantities [13].

\subsection{Relativistic invariance}

The total force on an object is often measured in experiment. However, this quantity is insufficient for determining the correct interpretations of electrodynamics as was demonstrated in the previous section. Moreover, the force densities differ between the various formulations [14], but it has been shown that many experimental observations can be modeled in the stationary limit (i.e. material velocity goes to zero $v \rightarrow 0$ ) by closing the system using a variety of subsystems [15]. In fact, the problem with the stationary approximation is the work-energy relation is always satisfied in a trivial manner [4]; $\bar{F} \cdot \bar{v}=\Phi=0$ since the velocity is zero in the limit. To determine which tensors are truly valid in a physical sense, we must look at relativistic invariance.

It is a fundamental tenet of modern physics that tensors which are not relativistic invariant cannot be energy momentum tensors. As an example, consider a region of space occupied by media described mathematically by a local mass density and a velocity field. The local momentum vector may vary with position and time regardless of how the coordinate system is assigned. Our inability to measure relativistic effects in any experiment may only be due to our limited measurement capabilities. This limitation does not prevent the fundamental laws of physics from holding [4].

For example, consider two frames of reference $\mathcal{S}$ and $\mathcal{S}^{\prime}$, whereas $\mathcal{S}^{\prime}$ moves with constant velocity $\bar{v}=\hat{x} v$ with respect to $\mathcal{S}$. The SEM tensor in $\mathcal{S}$ is

$$
\overline{\mathfrak{T}}_{j}=\left[\begin{array}{cccc}
\mathcal{T}_{x x} & \mathcal{T}_{x y} & \mathcal{T}_{x z} & i c G_{x} \\
\mathcal{T}_{y x} & \mathcal{T}_{y y} & \mathcal{T}_{y z} & i c G_{y} \\
\mathcal{T}_{z x} & \mathcal{T}_{z y} & \mathcal{T}_{z z} & i c G_{z} \\
i S_{x} / c & i S_{y} / c & i S_{z} / c & W
\end{array}\right]
$$

We want to know if the tensor is invariant under certain electrodynamic hypotheses. As a simple demonstration, we consider the Lorentz transform of the component

$$
\mathcal{T}_{x x}^{\prime}=\gamma^{2}\left[\mathcal{T}_{x x}+\beta\left(S_{x} / c+c G_{x}\right)-\beta^{2} W\right]
$$

where $\beta=v / c$ and $\gamma=(1-\beta)^{-1 / 2}$. To consider the five different hypotheses (i.e. Abraham, Minkowski, Amperian, Einstein-Laub, and Chu), we must consider the Lorentz transformation laws for the field variables within each field framework.

The Lorentz transformation laws for the Minkowski 
field variables $\bar{E}, \bar{H}, \bar{D}$, and $\bar{B}$ are [16]

$$
\begin{aligned}
\overline{\mathcal{E}}^{\prime} & =\overline{\mathcal{E}}_{\|}+\gamma\left(\overline{\mathcal{E}}_{\perp}+\bar{v} \times \overline{\mathcal{B}}\right), \\
\overline{\mathcal{H}}^{\prime} & =\bar{H}_{\|}+\gamma\left(\overline{\mathcal{H}}_{\perp}-\bar{v} \times \overline{\mathcal{D}}\right), \\
\overline{\mathcal{D}}^{\prime} & =\overline{\mathcal{D}}_{\|}+\gamma\left(\overline{\mathcal{D}}_{\perp}+\frac{\bar{v} \times \overline{\mathcal{H}}}{c^{2}}\right), \\
\overline{\mathcal{B}}^{\prime} & =\overline{\mathcal{B}}_{\|}+\gamma\left(\overline{\mathcal{B}}_{\perp}-\frac{\bar{v} \times \overline{\mathcal{E}}}{c^{2}}\right),
\end{aligned}
$$

where the subscripts $\|$ and $\perp$ denote parallel and perpendicular components to the velocity $\bar{v}$. These transformation laws are applied for the Abraham, Minkowski, and Einstein-Laub formulations, which utilize the Minkowski fields. The Lorentz transformation laws for the Chu field variables $\overline{\mathcal{E}}, \overline{\mathcal{H}}, \overline{\mathcal{P}}$, and $\overline{\mathcal{M}}$ are [16]

$$
\begin{aligned}
\overline{\mathcal{E}}^{\prime} & =\overline{\mathcal{E}}_{\|}+\gamma\left(\overline{\mathcal{E}}_{\perp}+\bar{v} \times \mu_{0} \overline{\mathcal{H}}\right) \\
\overline{\mathcal{H}}^{\prime} & =\overline{\mathcal{H}}_{\|}+\gamma\left(\overline{\mathcal{H}}_{\perp}-\bar{v} \times \epsilon_{0} \overline{\mathcal{E}}\right) \\
\overline{\mathcal{P}}^{\prime} & =\overline{\mathcal{P}}_{\|}+\gamma\left(\overline{\mathcal{P}}_{\perp}-\frac{\bar{v} \times(\overline{\mathcal{P}} \times \bar{v})}{c^{2}}\right) \\
\overline{\mathcal{M}}^{\prime} & =\overline{\mathcal{M}}_{\|}+\gamma\left(\overline{\mathcal{M}}_{\perp}-\frac{\bar{v} \times(\overline{\mathcal{M}} \times \bar{v})}{c^{2}}\right)
\end{aligned}
$$

where it is important to note that the Chu field values $\overline{\mathcal{E}}$ and $\overline{\mathcal{H}}$ differ from the Minkowski ones. We will make clear which field values are being applied within a given context. The Lorentz transformation laws for the Amperian field variables $\overline{\mathcal{E}}, \overline{\mathcal{B}}, \overline{\mathcal{P}}$, and $\overline{\mathcal{M}}$ are [16]

$$
\begin{aligned}
\overline{\mathcal{E}}^{\prime} & =\overline{\mathcal{E}}_{\|}+\gamma\left(\overline{\mathcal{E}}_{\perp}+\bar{v} \times \overline{\mathcal{B}}\right) \\
\overline{\mathcal{B}}^{\prime} & =\overline{\mathcal{B}}_{\|}+\gamma\left(\overline{\mathcal{B}}_{\perp}-\frac{\bar{v} \times \overline{\mathcal{E}}}{c^{2}}\right) \\
\overline{\mathcal{P}}^{\prime} & =\overline{\mathcal{P}}_{\|}+\gamma\left(\overline{\mathcal{P}}_{\perp}-\frac{\bar{v} \times(\overline{\mathcal{P}} \times \bar{v})}{c^{2}}\right) \\
\overline{\mathcal{M}}^{\prime} & =\overline{\mathcal{M}}_{\|}+\gamma\left(\overline{\mathcal{M}}_{\perp}-\frac{\bar{v} \times(\overline{\mathcal{M}} \times \bar{v})}{c^{2}}\right),
\end{aligned}
$$

Utilizing Eq. (9), we substitute the corresponding component values for $\bar{S}, W, \overline{\bar{T}}$, and $\bar{G}$ given in Tables 1 and 2 along with the field transformations for reference frame $\mathcal{S}$ to $\mathcal{S}^{\prime}$ using Eq. (10) for the Abraham, Minkowski, and Einstein-Laub tensors, Eq. (11) for the Chu tensor, and Eq. (12) for the Amperian tensor. After some algebraic manipulation, the results are summarized in Table 3. It can be seen that only the Minkowski, Chu, and Amperian SEM tensors retain their form after transformation. Such mathematical exercises can be carried-out on the remaining tensor components with similar results. The conclusion is that the Abraham and Einstein-Laub tensors are not valid SEM tensors [4].

\section{Energy and momentum in media}

\subsection{Field-kinetic subsystem}

The Minkowski, Amperian, and Chu SEM tensors differ in many regards, but one significant difference is the momen-
Table 3: Transformed stress tensors.

\begin{tabular}{lc}
\hline \hline & $\mathcal{T}_{x x}^{\prime}=-T_{x x}^{\prime}$ \\
\hline Abr. & $\mathcal{D}_{x}^{\prime} \mathcal{E}_{x}^{\prime}+\mathcal{B}_{x}^{\prime} \mathcal{H}_{x}^{\prime}-\frac{1}{2} \delta_{x x}\left(\overline{\mathcal{D}}^{\prime} \cdot \overline{\mathcal{E}}^{\prime}+\overline{\mathcal{B}}^{\prime} \cdot \overline{\mathcal{H}}^{\prime}\right)$ \\
& $+\gamma^{2} \beta\left[\epsilon_{0} \mu_{0}\left(\overline{\mathcal{E}}^{\prime} \times \overline{\mathcal{H}}^{\prime}\right)_{x}-\left(\overline{\mathcal{D}}^{\prime} \times \overline{\mathcal{B}}^{\prime}\right)_{x}\right]$ \\
\hline Min. & $\mathcal{D}_{x}^{\prime} \mathcal{E}_{x}^{\prime}+\mathcal{B}_{x}^{\prime} \mathcal{H}_{x}^{\prime}-\frac{1}{2} \delta_{x x}\left(\overline{\mathcal{D}}^{\prime} \cdot \overline{\mathcal{E}}^{\prime}+\overline{\mathcal{B}}^{\prime} \cdot \overline{\mathcal{H}}^{\prime}\right)$ \\
\hline Amp. $\quad \epsilon_{0} \mathcal{E}_{x}^{\prime} \mathcal{E}_{x}^{\prime}+\mu_{0}^{-1} \mathcal{B}_{x}^{\prime} \mathcal{B}_{x}^{\prime}-\frac{1}{2} \delta_{x x}\left(\epsilon_{0} \overline{\mathcal{E}}^{\prime} \cdot \overline{\mathcal{E}}^{\prime}+\mu_{0}^{-1} \overline{\mathcal{B}}^{\prime} \cdot \overline{\mathcal{B}}^{\prime}\right)$ \\
\hline E-L & $\gamma^{2}\left[\mathcal{E}_{x}^{\prime} \mathcal{D}_{x}^{\prime}+\mathcal{H}_{x}^{\prime} \mathcal{B}_{x}^{\prime}\right.$ \\
& $+2 \gamma^{2} \beta c^{-1}\left\{\left(\overline{\mathcal{E}}^{\prime} \times \overline{\mathcal{H}}^{\prime}\right)_{x}+c^{2} \beta^{2}\left(\overline{\mathcal{D}}^{\prime} \times \overline{\mathcal{B}}^{\prime}\right)_{x}\right.$ \\
& $\left.+c \beta\left(\mathcal{H}_{y}^{\prime} \mathcal{B}_{y}^{\prime}+\mathcal{H}_{z}^{\prime} \mathcal{B}_{z}^{\prime}+\mathcal{E}_{y}^{\prime} \mathcal{D}_{y}^{\prime}+\mathcal{E}_{z}^{\prime} \mathcal{D}_{z}^{\prime}\right)\right\}$ \\
& $-\frac{1}{2}\left(1+\beta^{2}\right)\left\{\epsilon_{0} \mathcal{E}_{x}^{\prime} \mathcal{E}_{x}^{\prime}+\mu_{0} \mathcal{H}_{x}^{\prime} \mathcal{H}_{x}^{\prime}\right.$ \\
& $+\gamma^{2}\left[\left(\epsilon_{0} \mathcal{E}_{y}^{\prime} \mathcal{E}_{y}^{\prime}+\epsilon_{0} \mathcal{E}_{z}^{\prime} \mathcal{E}_{z}^{\prime}+\mu_{0} \mathcal{H}_{y}^{\prime} \mathcal{H}_{y}^{\prime}+\mu_{0} \mathcal{H}_{z}^{\prime} \mathcal{H}_{z}^{\prime}\right)\right.$ \\
& $+c^{2} \beta^{2}\left(\epsilon_{0} \mathcal{B}_{y}^{\prime} \mathcal{B}_{y}^{\prime}+\epsilon_{0} \mathcal{B}_{z}^{\prime} \mathcal{B}_{z}^{\prime}+\mu_{0} \mathcal{D}_{y}^{\prime} \mathcal{D}_{y}^{\prime}+\mathcal{D}_{z}^{\prime} \mathcal{D}_{z}^{\prime}\right)$ \\
& $\left.\left.\left.+2 c \beta\left\{\left(\epsilon_{0} \overline{\mathcal{E}}^{\prime} \times \overline{\mathcal{B}}^{\prime}\right)_{x}+\left(\overline{\mathcal{D}}^{\prime} \times \mu_{0} \overline{\mathcal{H}}^{\prime}\right)_{x}\right\}\right]\right\}\right]$ \\
\hline Chu $\quad \epsilon_{0} \mathcal{E}_{x}^{\prime} \mathcal{E}_{x}^{\prime}+\mu_{0} \mathcal{H}_{x}^{\prime} \mathcal{H}_{x}^{\prime}-\frac{1}{2} \delta_{x x}\left(\epsilon_{0} \overline{\mathcal{E}}^{\prime} \cdot \overline{\mathcal{E}}^{\prime}+\mu_{0} \overline{\mathcal{H}}^{\prime} \cdot \overline{\mathcal{H}}^{\prime}\right)$ \\
\hline
\end{tabular}

tum density $\bar{G}$. While each of the three SEM tensors are relativistically invariant, it is desirable to determine if one of the three is the kinetic momentum of light, which is the momentum representing only field contributions without contributions from the mass of the material. In 1953, Balazs developed a thought experiment that allows for the determination of the kinetic momentum by studying the centerof-mass displacement of a material slab as an electromagnetic pulse passes [17], and number of researchers have presented versions of this thought experiment $[2,3,11,18,19]$.

We consider a slab of dispersive, impedance-matched material surrounded by vacuum. The slab is characterized by a group velocity $v_{g}=\partial \omega / \partial k$ and wave impedance is $\eta=\sqrt{\mu / \epsilon}=\sqrt{\mu_{0} / \epsilon_{0}}$. An electromagnetic wave pulse has an initial free space momentum $\mathfrak{E}_{i} / c$. The slab of thickness $d$ delays the pulse with respect to the free space path by the distance $L=\left(n_{g}-1\right) d$ since the group velocity in the material is $v_{g}=c / n_{g}$, where $n_{g}$ is the group velocity index of refraction. The required kinetic momentum of the material while the pulse overlaps spatially with the slab is

$$
p_{m}=\frac{\mathfrak{E}_{i}}{c}\left(1-\frac{1}{n_{g}}\right)
$$

which is necessary in order to maintain uniform motion of the center-of-mass energy. The momentum of the slab is the difference between the momentum of the incident pulse and the material momentum given by Eq. (13) as required by momentum conservation. Therefore, the electromagnetic momentum of the pulse is the field-kinetic momentum

$$
p_{F_{k}}=\frac{1}{n_{g}} \frac{\mathfrak{E}_{i}}{c} .
$$

This momentum corresponds to the Chu momentum, and we, therefore, take the Chu SEM tensor as the field-kinetic formulation [4]. Further discussion of this relationship is given in Section 4 of this correspondence. 
The Maxwell-Chu equations [3, 16, 20]

$$
\begin{aligned}
\nabla \times \overline{\mathcal{H}}-\epsilon_{0} \frac{\partial}{\partial t} \overline{\mathcal{E}} & =\overline{\mathcal{J}}_{e} \\
\nabla \times \overline{\mathcal{E}}+\mu_{0} \frac{\partial}{\partial t} \overline{\mathcal{H}} & =-\overline{\mathcal{J}}_{h} \\
\epsilon_{0} \nabla \cdot \overline{\mathcal{E}} & =\rho_{e} \\
\mu_{0} \nabla \cdot \overline{\mathcal{H}} & =\rho_{h}
\end{aligned}
$$

separate the electric and magnetic fields from the material response. The Chu formulation models the material responses by effective electric current density $\overline{\mathcal{J}}_{e}$, magnetic current density $\overline{\mathcal{J}}_{h}$, electric charge density $\rho_{e}$, and magnetic charge density $\rho_{h}$. These quantities for moving media with local velocity field $\bar{v}$ are defined as $[3,16,20]$

$$
\begin{aligned}
\overline{\mathcal{J}}_{e} & \equiv \frac{\partial \overline{\mathcal{P}}}{\partial t}+\nabla \times[\overline{\mathcal{P}} \times \bar{v}]+\overline{\mathcal{J}} \\
\overline{\mathcal{J}}_{h} & \equiv \mu_{0} \frac{\partial \overline{\mathcal{M}}}{\partial t}+\mu_{0} \nabla \times[\overline{\mathcal{M}} \times \bar{v}] \\
\rho_{e} & \equiv-\nabla \cdot \overline{\mathcal{P}}+\rho \\
\rho_{h} & \equiv-\mu_{0} \nabla \cdot \overline{\mathcal{M}},
\end{aligned}
$$

where $\overline{\mathcal{M}}$ is the magnetization, $\overline{\mathcal{P}}$ is the polarization, $\overline{\mathcal{J}}$ is the free current density, and $\rho$ is the free charge density of the given medium. The energy and momentum continuity equations for the field-kinetic subsystem are completed by the interaction terms $[3,16,20]$

$$
\begin{aligned}
\bar{f}_{F_{k}} & =\rho_{e} \overline{\mathcal{E}}+\rho_{h} \overline{\mathcal{H}}+\overline{\mathcal{J}}_{e} \times \mu_{o} \overline{\mathcal{H}}-\overline{\mathcal{J}}_{h} \times \epsilon_{0} \overline{\mathcal{E}}(17 \mathrm{a}) \\
\varphi_{F_{k}} & =\overline{\mathcal{J}}_{e} \cdot \overline{\mathcal{E}}+\overline{\mathcal{J}}_{h} \cdot \overline{\mathcal{H}}
\end{aligned}
$$

Next, we will derive the material response to the fieldkinetic subsystem for dielectrics and isotropic metamaterials with electric and magnetic response.

\subsection{Material response in dielectrics}

Consider a harmonic oscillator describing the motion of a bound charge under excitation of a time-harmonic field $\bar{E}$. The motion can be used to determine the stationary dielectric response of the charge $q$ given the damping factor $\gamma$ and the electric resonant frequency $\omega_{e 0}$. The equation of motion for $\bar{r}=\hat{x} x+\hat{y} y+\hat{z} z$ is [20]

$$
m\left(\frac{\partial^{2}}{\partial t^{2}}+\gamma_{e} \frac{\partial}{\partial t}+\omega_{e 0}^{2}\right) \bar{r}=q \overline{\mathcal{E}} .
$$

If there are $N$ such harmonic oscillators per volume of dielectric, the material subsystem is given by $[21,22,23]$

$$
\begin{aligned}
\bar{S}_{\text {mat }} & =0 \\
\bar{W}_{\text {mat }} & =N\left\{\frac{1}{2} m \frac{\partial \bar{r}}{\partial t} \cdot \frac{\partial \bar{r}}{\partial t}+\frac{1}{2} \omega_{e 0}^{2} m \bar{r} \cdot \bar{r}\right\} \\
\varphi_{\text {mat }} & =-\varphi_{F_{k}}+N\left\{\gamma_{e} \frac{\partial \bar{r}}{\partial t} \cdot \frac{\partial \bar{r}}{\partial t}\right\} .
\end{aligned}
$$

The energy terms consist of a kinetic energy term $\frac{1}{2} m \frac{\partial \bar{r}}{\partial t} \cdot \frac{\partial \bar{r}}{\partial t}$ and a potential energy term $\frac{1}{2} \omega_{0}^{2} m \bar{r} \cdot \bar{r}$ for each of $N$ charges in motion per-unit-volume. The momentum continuity equation for the material is [23]

$$
\begin{aligned}
\overline{\bar{T}}_{m a t} & =N\left\{\left[q \bar{r} \cdot \bar{E}+\frac{1}{2} m \frac{\partial \bar{r}}{\partial t} \cdot \frac{\partial \bar{r}}{\partial t}-\frac{1}{2} \omega_{e 0}^{2} m \bar{r} \cdot \bar{r}\right] \overline{\bar{I}}\right. \\
& -q \bar{r} \bar{E}\} \\
\bar{G}_{m a t} & =N\left\{q \bar{r} \times \mu_{0} \bar{H}-m \frac{\partial \bar{r}}{\partial t}\right\} \\
\bar{f}_{\text {mat }} & =-\bar{f}_{F_{k}}+N\left\{-\gamma_{e} m \frac{\partial \bar{r}}{\partial t}\right\} .
\end{aligned}
$$

Here, we recognize the terms proportional to $q \bar{r} \overline{\mathcal{E}}$ as the energy of the charge in the electric field, $\frac{1}{2} m|\partial \bar{r} / \partial t|^{2}$ as the kinetic energy of the particle, and $\frac{\omega_{e 0}^{2}}{2} m|\bar{r}|^{2}$ as the potential energy. This last term proportional to $\omega_{e 0}^{2}$ is due to the spring-like restoring nature between the electron and an oppositely charged nucleus, which is assumed to be much more massive.

Equation (18) can be written in terms of the macroscopic polarization definition by $\overline{\mathcal{P}}=N q \bar{r}$, giving the equation of motion as

$$
\left(\frac{\partial^{2}}{\partial t^{2}}+\gamma_{e} \frac{\partial}{\partial t}+\omega_{e 0}^{2}\right) \overline{\mathcal{P}}=\frac{N q^{2}}{m} \overline{\mathcal{E}}=\epsilon_{0} \omega_{e p}^{2} \overline{\mathcal{E}},
$$

where $\omega_{e p}=\sqrt{\frac{N q^{2}}{m \epsilon_{0}}}$ is the plasma frequency. The dielectric material contributions to the energy are $[1,3,6]$

$$
\begin{aligned}
\bar{S}_{\text {mat }} & =0 \\
\bar{W}_{\text {mat }} & =\frac{1}{2 \epsilon_{0} \omega_{e p}^{2}}\left[\frac{\partial \overline{\mathcal{P}}}{\partial t} \cdot \frac{\partial \overline{\mathcal{P}}}{\partial t}+\omega_{e 0}^{2} \overline{\mathcal{P}} \cdot \overline{\mathcal{P}}\right] \\
\varphi_{\text {mat }} & =-\varphi_{F_{k}}+\frac{\gamma}{\epsilon_{0} \omega_{e p}^{2}} \frac{\partial \overline{\mathcal{P}}}{\partial t} \cdot \frac{\partial \overline{\mathcal{P}}}{\partial t}
\end{aligned}
$$

The dielectric material contributions to the momentum are $[1,3,6]$

$$
\begin{aligned}
\overline{\bar{T}}_{m a t} & =\frac{1}{2}(\overline{\mathcal{P}} \cdot \overline{\mathcal{E}}) \overline{\bar{I}}-\overline{\mathcal{P}} \overline{\mathcal{E}} \\
& +\frac{1}{2}\left[\overline{\mathcal{P}} \cdot \overline{\mathcal{E}}+\frac{1}{\epsilon_{0} \omega_{e p}^{2}}\left(\frac{\partial \overline{\mathcal{P}}}{\partial t} \cdot \frac{\partial \overline{\mathcal{P}}}{\partial t}-\omega_{e 0}^{2} \overline{\mathcal{P}} \cdot \overline{\mathcal{P}}\right)\right] \overline{\bar{I}} \\
\bar{G}_{m a t} & =\overline{\mathcal{P}} \times \mu_{0} \overline{\mathcal{H}}-\frac{1}{\epsilon_{0} \omega_{e p}^{2}} \nabla \overline{\mathcal{P}} \cdot \frac{\partial \overline{\mathcal{P}}}{\partial t} \\
\bar{f}_{m a t} & =-\bar{f}_{F_{k}}-\frac{\gamma_{e}}{\epsilon_{0} \omega_{e p}^{2}} \nabla \overline{\mathcal{P}} \cdot \frac{\partial \overline{\mathcal{P}}}{\partial t} .
\end{aligned}
$$

The material subsystem and field-kinetic subsystem can be combined to form the canonical subsystem as described conceptually in Eq. (2).

\subsection{Energy and momentum in NIMs}

By duality of the field-kinetic (i.e Chu) subsystem, the causal dielectric and magnetic response of a stationary 
isotropic system can be formulated as [6, 24]

$$
\begin{array}{r}
\left(\frac{\partial^{2}}{\partial t^{2}}+\gamma_{e} \frac{\partial}{\partial t}+\omega_{e 0}^{2}\right) \overline{\mathcal{P}}=\epsilon_{0} \omega_{e p}^{2} \overline{\mathcal{E}} \\
\left(\frac{\partial^{2}}{\partial t^{2}}+\gamma_{m} \frac{\partial}{\partial t}+\omega_{m 0}^{2}\right) \mu_{0} \overline{\mathcal{M}}=\mu_{0} \omega_{m p}^{2} \overline{\mathcal{H}}
\end{array}
$$

The resulting material energy contributions are

$$
\begin{aligned}
\bar{S}_{m a t} & =0 \\
\bar{W}_{m a t} & =\frac{1}{2 \epsilon_{0} \omega_{e p}^{2}}\left[\frac{\partial \overline{\mathcal{P}}}{\partial t} \cdot \frac{\partial \overline{\mathcal{P}}}{\partial t}+\omega_{e 0}^{2} \overline{\mathcal{P}} \cdot \overline{\mathcal{P}}\right] \\
& +\frac{\mu_{0}}{2 \omega_{m p}^{2}}\left[\frac{\partial \overline{\mathcal{M}}}{\partial t} \cdot \frac{\partial \overline{\mathcal{M}}}{\partial t}+\omega_{m 0}^{2} \overline{\mathcal{P}} \cdot \overline{\mathcal{P}}\right] \\
\varphi_{m a t} & =-\varphi_{F_{k}}+\frac{\gamma_{e}}{\epsilon_{0} \omega_{e p}^{2}} \frac{\partial \overline{\mathcal{P}}}{\partial t} \cdot \frac{\partial \overline{\mathcal{P}}}{\partial t} \\
& +\frac{\gamma_{m}}{\epsilon_{0} \omega_{m p}^{2}} \frac{\partial \overline{\mathcal{M}}}{\partial t} \cdot \frac{\partial \overline{\mathcal{M}}}{\partial t},
\end{aligned}
$$

and the momentum contributions are

$$
\begin{aligned}
\overline{\bar{T}}_{m a t} & =\frac{1}{2}\left(\overline{\mathcal{P}} \cdot \overline{\mathcal{E}}+\mu_{0} \overline{\mathcal{M}} \cdot \overline{\mathcal{H}}\right) \overline{\bar{I}}-\overline{\mathcal{P}} \overline{\mathcal{E}}-\mu_{0} \overline{\mathcal{M}}(\overline{\text { Zb }} \mathrm{a}) \\
& +\frac{1}{2}\left\{\overline{\mathcal{P}} \cdot \overline{\mathcal{E}}+\mu_{0} \overline{\mathcal{M}} \cdot \overline{\mathcal{H}}\right. \\
& +\frac{1}{\epsilon_{0} \omega_{e p}^{2}}\left(\frac{\partial \overline{\mathcal{P}}}{\partial t} \cdot \frac{\partial \overline{\mathcal{P}}}{\partial t}-\omega_{e 0}^{2} \overline{\mathcal{P}} \cdot \overline{\mathcal{P}}\right) \\
& \left.+\frac{\mu_{0}}{\omega_{m p}^{2}}\left(\frac{\partial \overline{\mathcal{M}}}{\partial t} \cdot \frac{\partial \overline{\mathcal{M}}}{\partial t}-\omega_{m 0}^{2} \overline{\mathcal{M}} \cdot \overline{\mathcal{M}}\right)\right\} \overline{\bar{I}} \\
\bar{G}_{m a t} & =\overline{\mathcal{P}} \times \mu_{0} \overline{\mathcal{H}}+\overline{\mathcal{E}} \times \mu_{0} \overline{\mathcal{M}}+\overline{\mathcal{P}} \times \mu_{0} \overline{\mathcal{M}} \\
& -\frac{1}{\epsilon_{0} \omega_{e p}^{2}} \nabla \overline{\mathcal{P}} \cdot \frac{\partial \overline{\mathcal{P}}}{\partial t}-\frac{\mu_{0}}{\omega_{m p}^{2}} \nabla \overline{\mathcal{M}} \cdot \frac{\partial \overline{\mathcal{M}}}{\partial t} \quad \text { (26b) } \\
\bar{f}_{m a t} & =-\bar{f}_{F_{k}}-\frac{\gamma_{e}}{\epsilon_{0} \omega_{e p}^{2}} \nabla \overline{\mathcal{P}} \cdot \frac{\partial \overline{\mathcal{P}}}{\partial t} \\
& -\frac{\mu_{0} \gamma_{m}}{\omega_{m p}^{2}} \nabla \overline{\mathcal{M}} \cdot \frac{\partial \overline{\mathcal{M}}}{\partial t} .
\end{aligned}
$$

Under time-harmonic excitation, the stationary (i.e. $\bar{v} \rightarrow 0$ ) Minkowski constitutive parameters can be approximated $\bar{D}=\epsilon(\omega) \bar{E}$ and $\bar{B}=\mu(\omega) \bar{H}$. Each field phaser follows the relation $\overline{\mathcal{A}}=\Re\left\{\bar{A} e^{-i \omega t}\right\}$. The constitutive parameters are

$$
\begin{aligned}
& \epsilon(\omega)=\epsilon_{0}\left(1-\frac{\omega_{e p}^{2}}{\omega^{2}-\omega_{e 0}^{2}+i \omega \gamma_{e}}\right) \\
& \mu(\omega)=\mu_{0}\left(1-\frac{\omega_{m p}^{2}}{\omega^{2}-\omega_{m 0}^{2}+i \omega \gamma_{m}}\right) .
\end{aligned}
$$

The time-averaged energy density is

$$
\begin{aligned}
\left\langle W_{c a n}\right\rangle & =\frac{\epsilon_{0}}{2}\left[1+\frac{\omega_{e p}^{2}\left(\omega^{2}+\omega_{e 0}^{2}\right)}{\left(\omega^{2}-\omega_{e 0}^{2}\right)^{2}+\gamma_{e}^{2} \omega^{2}}\right]|\bar{E}|^{2} \\
& +\frac{\mu_{0}}{2}\left[1+\frac{\omega_{m p}^{2}\left(\omega^{2}+\omega_{m 0}^{2}\right)}{\left(\omega^{2}-\omega_{m 0}^{2}\right)^{2}+\gamma_{m}^{2} \omega^{2}}\right]|\bar{H}|^{2}(28)
\end{aligned}
$$

In the limit of zero losses $\left(\gamma_{e} \rightarrow 0\right.$ and $\left.\gamma_{m} \rightarrow 0\right)$ both $\epsilon_{I}=0$ and $\mu_{I}=0$ and the energy density satisfies the well-known relation $[20,21,25,26]$

$$
\left\langle W_{\text {can }}\right\rangle=\frac{1}{4} \frac{\partial(\epsilon \omega)}{\partial \omega}|\bar{E}|^{2}+\frac{1}{4} \frac{\partial(\mu \omega)}{\partial \omega}|\bar{H}|^{2} .
$$

It is the rate of change in energy that appears in the energy continuity equation, which tends to zero upon cycle averaging, and since $\langle\partial W / \partial t\rangle=0$, the resulting conservation equation

$$
-\left\langle\nabla \cdot \bar{S}_{c a n}\right\rangle=\frac{1}{2}\left[\omega \epsilon_{I}|\bar{E}|^{2}+\omega \mu_{I}|\bar{H}|^{2}\right]
$$

is generally regarded as the complex Poynting's theorem, where $\left\langle\bar{S}_{c a n}\right\rangle=\frac{1}{2} \Re\left\{\bar{E} \times \bar{H}^{*}\right\}$ is the time average Poynting power.

The average momentum density,

$$
\begin{aligned}
\left\langle\bar{G}_{c a n}\right\rangle & =\frac{1}{2} \Re\left\{\bar{D} \times \bar{B}^{*}+\bar{k} \frac{\epsilon_{0} \omega \omega_{e p}^{2}}{\left(\omega^{2}-\omega_{e 0}^{2}\right)^{2}+\gamma_{e}^{2} \omega^{2}}|\bar{E}|^{2}\right. \\
& \left.+\bar{k} \frac{\mu_{0} \omega \omega_{m p}^{2}}{\left(\omega^{2}-\omega_{m 0}^{2}\right)^{2}+\gamma_{m}^{2} \omega^{2}}|\bar{H}|^{2}\right\}
\end{aligned}
$$

satisfies [27]

$$
\begin{aligned}
\left\langle\bar{G}_{\text {can }}\right\rangle & =\frac{1}{2} \Re\left\{\bar{D} \times \bar{B}^{*}+\frac{\bar{k}}{2}\left(\frac{\partial \epsilon}{\partial \omega}|\bar{E}|^{2}+\frac{\partial \mu}{\partial \omega}|\bar{H}|^{2}\right)\right\} \\
& =\frac{1}{2}\left\{\frac{1}{v_{g} v_{p}} \bar{E} \times \bar{H}^{*}\right\}
\end{aligned}
$$

when the losses approach zero, where $v_{p}=\omega / k$ and $v_{g}=\partial \omega / \partial k$ are the phase velocity and the group velocity, respectively. The time average stress tensor is found to be

$$
\left\langle\overline{\bar{T}}_{c a n}\right\rangle=\frac{1}{2} \Re\left\{\frac{1}{2}\left(\bar{D} \cdot \bar{E}^{*}+\bar{B} \cdot \bar{H}^{*}\right) \overline{\bar{I}}-\bar{D} \bar{E}^{*}-\bar{B} \bar{H}^{*}\right\}
$$

since the dispersive terms average to zero. Because the average rate of change in momentum density is zero (i.e. $\langle\partial \bar{G} / \partial t\rangle=0$ ), the momentum conservation theorem for a monochromatic wave becomes

$$
-\left\langle\nabla \cdot \overline{\bar{T}}_{c a n}\right\rangle=\frac{1}{2} \Re\left\{\omega \epsilon_{I} \bar{E} \times \bar{B}^{*}-\omega \mu_{I} \bar{H} \times \bar{D}^{*}\right\}
$$

where the tensor is the complex Minkowski tensor. The force terms in Eq. (34) have been defined as the force density on free currents $[28,29]$.

\section{Discussion}

For illustration purposes, we consider a couple of examples. First, we consider the momentum density contribution to an electromagnetic pulse. Then, we consider the momentum flux or stress tensor contribution to a continuous wave. 


\subsection{Electromagnetic Pulse}

A pulse is incident upon a medium with constitutive parameters $\mu(\omega), \epsilon(\omega)$. For simplicity, we consider the medium to be dispersive, but lossless. The excitation energy of the pulse is $[1,3]$

$$
\mathfrak{E}_{e x c} \equiv \mathfrak{E}_{i}-\mathfrak{E}_{r}=\mathfrak{E}_{i}\left(1-|R|^{2}\right),
$$

where $\mathfrak{E}_{i}$ is the incident energy, $\mathfrak{E}_{r}$ is the reflected energy, and $|R|^{2}$ is the reflectivity. The excitation energy is unambiguously defined in terms of the vacuum quantities. The spatial length of the transmitted pulse is decreased proportional to the factor $n_{g}^{-1}$ due to the change in velocity inside the medium. We can investigate the interpretation of the field-kinetic and canonical subsystems inside the medium.

If we assume the field-kinetic momentum density defined by the Chu SEM tensor, we see that the momentum inside the material is

$$
\begin{aligned}
\bar{p}_{F_{k}} & =\int_{z} d z \bar{G}_{F_{k}}(z)=\hat{z} \frac{1}{n_{g}} \frac{\mathfrak{E}_{i}}{c}\left(1-R^{2}\right) \\
& =\hat{z} \frac{1}{n_{g} c} \mathfrak{E}_{\text {exc }} .
\end{aligned}
$$

This is the momentum derived in Balazs' thought experiment.

We may arrive at this result using another approach by viewing each photon as a particle with effective mass unchanged as it enters the material so that $m_{0}=m=\mathfrak{E} / c^{2}$. In the vacuum region $(z<0)$, the velocity of the pulse is $\bar{v}_{0}=\hat{z} c$. At $t=0$, the pulse enters the material and the velocity becomes $\bar{v}=\hat{z} c / n_{g}$. The force on the material is due to the momentum exchange

$$
\begin{aligned}
\bar{p}_{i}-\bar{p}_{k i n} & =m_{0} \bar{v}_{0}-m \bar{v}=\hat{z}\left(\frac{\mathfrak{E}}{c^{2}} c-\frac{\mathfrak{E}}{c^{2}} \frac{c}{n_{g}}\right) \\
& =\hat{z} \frac{\mathfrak{E}}{c}\left(1-\frac{1}{n_{g}}\right) .
\end{aligned}
$$

Therefore, the field-kinetic subsystem gives the interpretation of constant effective mass.

Alternately, if we assume the canonical momentum density defined for a dispersive, lossless material from Eq. (32)

$$
\bar{G}_{c a n}(z)=\hat{z} n_{g} n_{p} \frac{W_{i}(z)}{c}\left(1-|R|^{2}\right),
$$

we see that the momentum inside the material is

$$
\begin{aligned}
\bar{p}_{c a n} & =\int_{z} d z \bar{G}_{c a n}(z)=\hat{z} n_{p} \frac{\mathfrak{E}_{i}}{c}\left(1-R^{2}\right) \\
& =\hat{z} n_{p} \frac{\mathfrak{E}_{e x c}}{c} .
\end{aligned}
$$

This momentum, which is proportional to the phase velocity index of refraction, is generally measured inside materials as a combination of field plus material contributions [12, 28, 30, 31, 32].

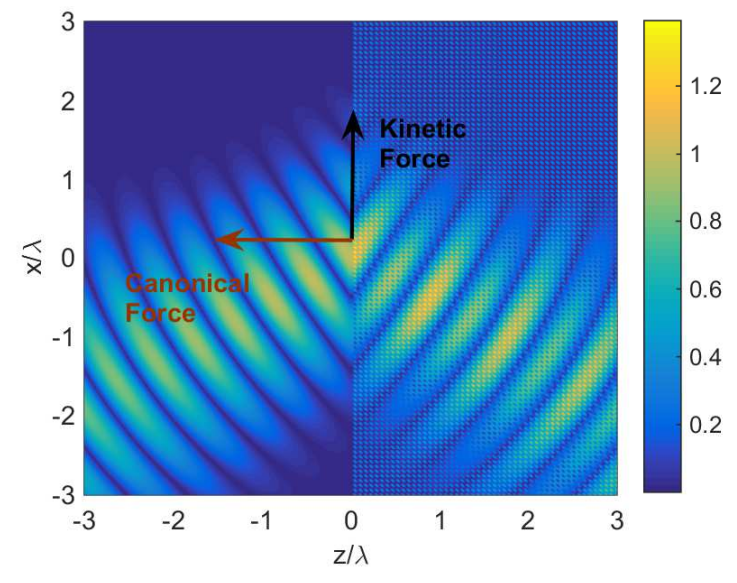

Figure 2: Demonstration of a refracted Gaussian beam at a matched negative refractive index boundary. The incident medium $z<0$ is vacuum $\left(\mu_{0}, \epsilon_{0}\right)$ and the transmitted is a matched left-handed medium $\left(-\epsilon_{0},-\mu_{0}\right)$. The parameters for incident TM field are amplitude $H_{0}=1 \mathrm{~A} / \mathrm{m}$, incident wavelength $\lambda_{0}=1064 \mathrm{~nm}$, beam waist $w=\lambda_{0}$, and $N A=0.8$

We may arrive at this result using another approach by viewing each photon as a particle where the effective mass increases when it enters the material. The force on the material is due to the momentum exchange

$$
\begin{aligned}
\bar{p}_{i}-\bar{p}_{c a n} & =m_{0} \bar{v}_{0}-m \bar{v}=\hat{z}\left(\frac{\mathfrak{E}}{c^{2}} c-\frac{\mathfrak{E}}{c^{2} /\left(n_{g} n_{p}\right)} \frac{c}{n_{g}}\right) \\
& =\hat{z} \frac{\mathfrak{E}}{c}\left(1-n_{p}\right) .
\end{aligned}
$$

In this view, the momentum of the pulse entering a dielectric is increased due to the additional momentum of the material which is added to the electromagnetic pulse. These contributions were described in detail in Section 3.

It is interesting what happens to the two quantities in negative index media (NIM). Note that for NIM, $n_{p}<0$, but $n_{g}>0$. The field-kinetic momentum, being related to $n_{g}$, remains positive in negative index media. The canonical momentum on the other hand reverses direction due to its proportionality to $n_{p}$. Note, however that in the presence of losses, the direction of the canonical momentum can be parallel or anti-parallel to the energy flow [6].

\subsection{Continuous Wave}

Consider the problem of a continuous wave incident upon a negative index material $\left(-\epsilon_{0},-\mu_{0}\right)$ from vacuum $\left(\epsilon_{0}, \mu_{0}\right)$. The wave shown in Fig. 2 is a TM Gaussian beam incident at $\pi / 6$.

The field-kinetic force on the surface is $\bar{F}_{F_{k}}=$ $\hat{x} 1.6 \mathrm{pN} / \mathrm{m}$. The interpretation of this force is that there is an equal and opposite force $-\bar{F}_{F_{k}}=-\hat{x} 1.6 \mathrm{~N} / \mathrm{m}$ that changes the direction of the incident photons from the $+\hat{z}$ and $+\hat{x}$ direction to the $+\hat{z}$ and $-\hat{x}$ direction. The transmitted electromagnetic momentum has an identical $\hat{z}$ com- 
ponent as the incident. Therefore, it is the equal and opposite force of the material on the electromagnetic waves at the surface that causes the change in momentum in the $\hat{x}$ direction. The form of the field-kinetic momentum remains the same for all types of medium, and this description was previously applied to describe the optical dynamics of anisotropic metamaterial cloaks [33, 34]. Although the field-kinetic subsystem had yet to be uniquely identified, the Chu formulation was used to describe the optical dynamics of optical cloaks, which explains in retrospect how the forces on the optical paths were able to describe the transformed paths. Such a description is also useful for perfect lenses where planar surfaces are used to negatively refract images [24].

The canonical SEM tensor provides an alternate viewpoint. In this case, the time-averaged force reduces to the divergence of the Minkowski stress tensor. The canonical force on the surface is $\bar{F}_{\text {can }}=\hat{z} 2.8 \mathrm{pN} / \mathrm{m}$. The zero force in the $\hat{x}$ direction means that the tangential component of the wave momentum is conserved [6]. The change in the normal component of the wave momentum is due to the additional momentum from the material response as described in Section 3.

\section{Conclusions}

We have shown that the field-kinetic subsystem as given by the Chu formulation represents the energy and momentum contained within the fields, and the sum of the field-kinetic and material response subsystems represent the wave or canonical subsystem. The canonical subsystem reduces to the Minkowski subsystem only under negligible dispersion. We may conclude that neither the Minkowski or Abraham SEM tensors are universally correct. In fact, the Abraham SEM tensor violates the physical constraint of relativistic invariance. Our view was demonstrated the physics of negative index materials under both the field-kinetic and canonical or wave SEM models for negative refraction, which is fundamental to perfect lensing and invisibility cloaking.

\section{Acknowledgement}

This work was sponsored in part by the NSF Division of Electrical, Communications, and Cyber Systems (Award No. ECCS-1150514) through the Faculty Early Career Development (CAREER) program, the Arkansas EPSCoR program provided by the National Science Foundations Research Infrastructure Improvement (Award No. OIA-1457888), and the Arkansas Research Alliance Fellows program.

\section{References}

[1] B. A. Kemp, "Resolution of the Abraham-Minkowski debate: Implications for the electromagnetic wave theory of light in matter," J. Appl. Phys., vol. 109, p. 111101, 2011.
[2] S. M. Barnett, "Resolution of the AbrahamMinkowski dilemma," Phys. Rev. Lett., vol. 104, p. 070401, 2010.

[3] B. A. Kemp, "Macroscopic theory of optical momentum," Prog. Optics, vol. 60, p. 437, 2015.

[4] C. J. Sheppard and B. A. Kemp, "Kinetic-energymomentum tensor in electrodynamics," Phys. Rev. A, vol. 93, p. 013855, 2016.

[5] C. J. Sheppard and B. A. Kemp, "Relativistic analysis of field-kinetic and canonical electromagnetic systems," Phys. Rev. A, vol. 93, p. 053832, 2016.

[6] B. A. Kemp, J. A. Kong, and T. M. Grzegorczyk, "Reversal of wave momentum in isotropic left-handed media," Phys. Rev. A, vol. 75, p. 053810, 2007.

[7] T. M. Grzegorczyk and B. A. Kemp, "Transfer of optical momentum: reconciliations of the abraham and minkowski formulations," Proc. SPIE, vol. 7038, p. 70381S, 2008.

[8] M. Mansuripur, "Radiation pressure and the linear momentum of the electromagnetic field," Opt. Express, vol. 12, p. 5375, 2004.

[9] B. A. Kemp, T. M. Grzegorczyk, and J. A. Kong, "Lorentz force on dielectric and magnetic particles," J. of Electromagn. Waves and Appl., vol. 20, p. 827, 2006.

[10] B. A. Kemp and T. M. Grzegorczyk, "The observable pressure of light in dielectric fluids," Opt. Lett., vol. 36, no. 4, p. 493, 2011.

[11] C. J. Sheppard and B. A. Kemp, "Optical pressure deduced from energy relations within relativistic formulations of electrodynamics," Phys. Rev. A, vol. 89, no. 1, p. $013825,2014$.

[12] B. A. Kemp, "Subsystem approach to the electrodynamics in dielectric fluids," Proc. SPIE, vol. 8458, p. 845803, 2012.

[13] B. A. Kemp, "Comment on "revisiting the balazs thought experiment in the presence of loss: electromagnetic-pulse-induced displacement of a positive-index slab having arbitrary complex permittivity and permeability"," Appl. Phys. A, vol. 110, p. 517, 2013.

[14] M. Mansuripur, A. R. Zakharian, and E. W. Wright, "Electromagnetic-force distribution inside matter," Phys. Rev. A, vol. 88, p. 023826, 2013.

[15] N. G. C. Astrath, L. C. Malacarne, M. L. Baesso, G. V. B. Lukasievicz, and S. E. Bialkowski, "Unravelling the effects of radiation forces in water," Nat. Commun., vol. 5, p. 4363, 2014. 
[16] P. Penfield and H. A. Haus, Electrodynamics of Moving Media. Cambridge, MA: M.I.T. Press, 1967.

[17] N. L. Balazs, Phys. Rev., vol. 91, p. 408, 195d.

[18] R. Loudon, "Radiation pressure and momentum in dielectrics," Fortschr. Phys, vol. 52, p. 1134, 2004.

[19] M. G. Scullion and S. M. Barnett, "Optical momentum in negative-index media," J. Mod. Opt., vol. 55, p. 2301, 2008.

[20] J. A. Kong, Electromagnetic Wave Theory. Cambridge, MA: EMW Publishing, 2005.

[21] L. Brillouin, Wave Propagation and Group Velocity. New York: Academic Press, 1960.

[22] R. Loudon, "The propagation of electromagnetic energy through an absorbing dielectric," J. Phys. A, vol. 3, p. 233, 1970.

[23] R. Loudon, L. Allen, and D. F. Nelson, "Propagation of electromagnetic energy and momentum through an absorbing dielectric," Phys. Rev. E, vol. 55, p. 1071, 1997.

[24] S. A. Ramakrishna and T. M. Grzegorczyk, Physics and Applications of Negative Refractive Index Materials. CRC Press, 2008.

[25] L. D. Landau, E. M. Lifshitz, and L. P. Pitaevskii, Electrodynamics of Continuous Media, 2nd ed. New York: Pergamon, 1984.

[26] J. D. Jackson, Classical Electrodynamics. New York: John Wiley and Sons, 1999.

[27] V. G. Veselago, "The electrodynamics of substances with simultaneously negative values of $\epsilon$ and $\mu$," Sov. Phys. Usp., vol. 10, p. 509, 1968.

[28] R. Loudon, S. M. Barnett, and C. Baxter, "Radiation pressure and momentum transfer in dielectrics: The photon drag effect," Phys. Rev. A, vol. 71, p. 063802, 2005.

[29] B. A. Kemp, T. M. Grzegorczyk, and J. A. Kong, "Optical momentum transfer to absorbing Mie particles," Phys. Rev. Lett., vol. 97, p. 133902, 2006.

[30] R. V. Jones and J. C. S. Richards, "The pressure of radiation in a refracting medium," Proc. R. Soc. Lond. A., vol. 221, p. 480, 1954.

[31] R. V. Jones and B. Leslie, "The measurement of optical radiation pressure in dispersive media," Proc. $R$. Soc. Lond. A., vol. 360, p. 347, 1978.

[32] G. K. Campbell, A. E. Leanhardt, J. Mun, M. Boyd, E. W. Streed, W. Ketterle, and D. E. Pritchard, "Photon recoil momentum in dispersive media," Phys. Rev. Lett., vol. 94, p. 170403, 2005.
[33] H. Chen, B. Zhang, Y. Luo, B. A. Kemp, J. Zhang, L. Ran, and B.-I. Wu, "Lorentz force and radiation pressure on a spherical cloak," Phys. Rev. A, vol. 80, p. 011808, 2009.

[34] H. Chen, B. Zhang, B. A. Kemp, and B.-I. Wu, "Optical force on a cylindrical cloak under arbitrary wave illumination," Opt. Lett., vol. 35, p. 667, March 2010. 